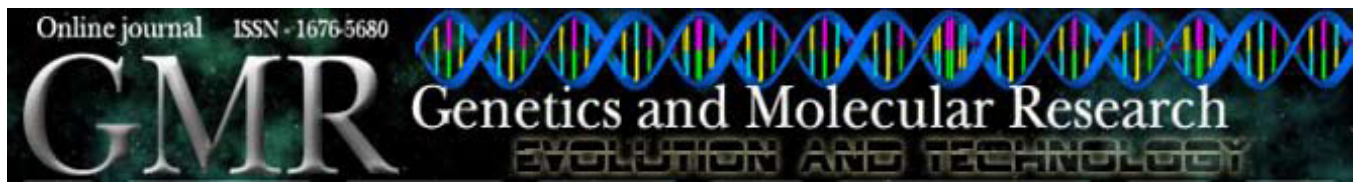

\title{
mRNA abundance and expression of SLC27A, ACC, SCD, FADS, LPIN, INSIG, and PPARGC1 gene isoforms in mouse mammary glands during the lactation cycle
}

\author{
L.Q. Han ${ }^{1,2}$, H.J. Li ${ }^{1}$, Y.Y. Wang ${ }^{1}$, H.S. Zhu' ${ }^{1}$, L.F. Wang ${ }^{1}$, Y.J. Guo ${ }^{1}$, \\ W.F. Lu ${ }^{1}$, Y.L. Wang ${ }^{2}$ and G.Y. Yang ${ }^{1,2}$ \\ ${ }^{1}$ College of Animal Science and Veterinary Medicine, \\ Henan Agricultural University, Zhengzhou, China \\ ${ }^{2}$ Key Laboratory of Animal Growth and Development, \\ Ministry of Agriculture, Zhengzhou, China \\ Corresponding author: G.Y. Yang \\ E-mail:mrswx@yahoo.cn
}

Genet. Mol. Res. 9 (2): 1250-1257 (2010)

Received March 4, 2010

Accepted April 3, 2010

Published June 29, 2010

DOI 10.4238/vol9-2gmr814

\begin{abstract}
The functions of distinct isoforms of solute carrier family 27 transporters (SLC27A1-6), acetyl-CoA carboxylase (ACACA, ACACB), stearoyl-CoA desaturase (SCD1-4), fatty acid desaturase (FADS1-3), LPIN (LPIN1-3), insulin-induced gene (INSIG1, 2), and peroxisome proliferator-activated receptor gamma coactivator1 (PPARGC1A, B) were studied in the mouse mammary gland from pregnancy to lactation. The relative mRNA abundance and percent change in real-time PCR were determined. mRNA expression of SLC27A3 and SLC27A4 was 37- and 1.4-fold more upregulated at 12 days of lactation, respectively $(\mathrm{P}<0.01)$. Transcripts of SCD isoforms were the most abundant, accounting for 59\% of all genes measured, and PPARGC1 isoforms were the least $(0.06 \%$ of all genes measured). The mRNA abundance from ACC, FADS and LPIN accounted for 29, 9 and 2.6\%, respectively. INSIG1 mRNA expression was 32-fold more upregulated ( $\mathrm{P}<0.05$ ), while PPARGC1B was 0.18 fold downregulated at 18 days of lactation $(\mathrm{P}<0.01)$. We concluded that
\end{abstract}


mRNA abundance and expression of these isoforms are affected by the stage of lactation.

Key words: Isoform; Lactation; Lipogenic gene; Mouse mammary gland; Quantitative real-time PCR

\section{INTRODUCTION}

The mammary gland of mouse is estimated to produce an extraordinary $32 \mathrm{~g}$ triacylglyceride (TAG) over a lactation period of just 20 days, making it perhaps the most active lipid-secreting organ known (Schwertfeger et al., 2003). Some research has shown a crucial role for the gene expression of key proteins (transporters, enzymes, regulators, etc.) involved in TAG synthesis in the mammary gland during lactation (Rudolph et al., 2007; Bernard et al., 2008; Bionaz and Loor, 2008a), but the existence of several uncharacterized isoforms in the mouse makes this conclusion unreliable (Rudolph et al., 2007).

The isoforms of these genes have different actions, but few studies have been conducted to relate the gene expression patterns in the mammary gland. Nevertheless, thanks to the development of molecular biology tools, mRNA abundance and expression of these isoforms could be calculated based on their real-time polymerase chain reaction (PCR) efficiency and the crossing point difference $(\Delta \mathrm{Ct})$ (Bionaz and Loor, 2008b).

The aim of the present study was to investigate the mRNA abundance and expression of lipogenic genes, mainly considered the genes for the fatty acid transporter of solute carrier family 27 transporters (SLC27A1-6), fatty acid synthesis enzymes of acetyl-CoA carboxylase (ACACA, ACACB), stearoyl-CoA desaturase (SCD1-4), fatty acid desaturase (FADS1-3), LPIN (LPIN1-3), regulator of insulin-induced gene (INSIG1, 2), and peroxisome proliferator-activated receptor gamma coactivator1 (PPARGC1A, B), involved in the TAG synthesis in mouse mammary glands throughout the lactation cycle. The measurement of these isoforms will provide valid information for better understanding TAG synthesis in the mammary gland.

\section{MATERIAL AND METHODS}

\section{Animals and sampling}

Thirty-two KM pregnant mice were supplied by the laboratory animal research central of Zhengzhou Medical University. P1 was identified as the first day when a postcoital plug was observed. L1 was identified as the first day when litters were present. The litter number was normalized to 8 on L3. Mammary glands from eight mice were taken for the time series at P18 and L6, L12, and L18. The excised gland was frozen in liquid nitrogen.

\section{Primer design}

Reference genes (GAPDH, HPRT and RPL13A) and target genes (SLC27A, ACC, SCD, FADS, LPIN, INSIG, and PPARGC1) were selected for evaluation throughout the different de- 
velopmental stages. Primer sequences were designed with the Primer Express Software (Applied Biosystems). The primer sequence, correlation of stand curve and reaction efficiencies for the genes were submitted to the RT Primer Database [http://medgen.ugent.be/rtprimerdb/] and are shown in Supplementary Table S1.

\section{RNA extraction and qRT-PCR}

Total RNA was extracted from $100 \mathrm{mg}$ mammary tissue using the Pure Yield TMRNA Midiprep System (Promega) according to the instruction manual. Each cDNA was synthesized with M-MLV reverse transcriptase (Promega) using equivalent amounts of total RNA ( $2 \mu \mathrm{g})$. Real-time PCR was performed in an Eppendorf Mastercyler realplex instrument. The $\mathrm{Ct}$ value was calculated using the Instrument software.

\section{Percent mRNA abundance among isoforms}

The efficiency of PCR application for each gene was calculated with the standard curve method $\left(\mathrm{E}=10^{-(1 / \log \text { CURVE SLOPE) }}\right)$. Relative mRNA abundance among all genes measured was calculated as reported by Bionaz and Loor (2008b), using the inverse of PCR efficiency raised to the power $\Delta \mathrm{Ct}$ (gene abundance $=1 / \mathrm{E}^{\Delta \mathrm{Ct}}$, where $\Delta \mathrm{Ct}=\mathrm{Ct}$ sample-geometric mean $\mathrm{Ct}$ of 3 reference genes). The percent mRNA abundance for each isoform gene among all genes measured was calculated using the median $\Delta \mathrm{Ct}$. The percent abundance of each isoform for each time evaluated (P18, L6, L18 and L12) was calculated using the median $\Delta \mathrm{Ct}$ of each time. The description of the genes measured and overall \% relative mRNA abundance are shown in Table 1.

\section{Fold change in mRNA expression among isoforms}

Normalized quantitative real-time PCR data were presented as fold change relative to P18. To calculate the relative expression ratio of the target gene, we used the formula described by Pfaffl (2001) and Vandesompele et al. (2002):

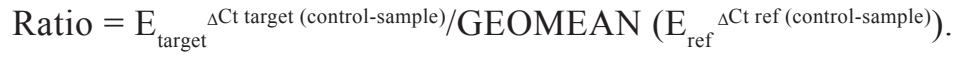

The data were analyzed using an ANOVA model in SPSS10.0.

\section{RESULTS}

Overall, SLC27A isoform mRNA abundance represented about $1 \%$ of all genes measured (Table 1). SLC27A4 mRNA accounts for $0.59 \%$, followed by mRNA of SLC27A3 (0.24\%). SLC27A1 mRNA was detected in lower abundance (0.1\%). Other isoforms were present in trace amounts, and isoform 5 was undetectable (Table 1). The mRNA expression of SLC27A4 and SLC27A3 was affected by the stage of lactation and showed a tendency to increase 1.4- and 37-fold at L12, respectively ( $\mathrm{P}<$ 0.01 ; Figure 1), while mRNA expression of other SLC27A isoforms did not vary significantly during the lactation cycle. 
Table 1. Relative mRNA abundance of isoforms (\%) during the lactation cycle.

\begin{tabular}{|c|c|c|c|c|c|}
\hline Gene & $\mathrm{P} 18^{\mathrm{a}}$ & L6 & L12 & L18 & Overall mRNA abundance ${ }^{\mathrm{b}}$ \\
\hline SLC27A1 & 34.19 & 6.09 & 7.67 & 9.12 & 0.10 \\
\hline SLC27A2 & 3.27 & 0.40 & 0.14 & 0.37 & $<0.01$ \\
\hline SLC27A3 & 1.72 & 23.85 & 47.69 & 39.90 & 0.24 \\
\hline SLC27A4 & 59.75 & 67.61 & 44.24 & 50.12 & 0.59 \\
\hline SLC27A5 & - & - & - & - & - \\
\hline SLC27A6 & 1.04 & 2.03 & 0.23 & 0.47 & $<0.01$ \\
\hline ACACA & 99.94 & 99.96 & 99.95 & 99.95 & 29.63 \\
\hline $\mathrm{ACACB}$ & 0.05 & 0.03 & 0.04 & 0.04 & 0.01 \\
\hline SCD1 & 74.03 & 70.84 & 70.39 & 67.48 & 42.34 \\
\hline SCD2 & 25.96 & 29.15 & 29.60 & 32.51 & 17.50 \\
\hline SCD3 & $<0.01$ & $<0.01$ & $<0.01$ & $<0.01$ & $<0.01$ \\
\hline SCD4 & $<0.01$ & $<0.01$ & $<0.01$ & $<0.01$ & $<0.01$ \\
\hline FADS1 & 92.09 & 69.92 & 70.86 & 73.39 & 7.56 \\
\hline FADS2 & 7.90 & 30.07 & 29.13 & 26.60 & 1.98 \\
\hline FADS3 & - & - & - & - & - \\
\hline LPIN1 & 87.48 & 91.59 & 93.58 & 93.71 & 2.50 \\
\hline LPIN2 & 12.51 & 8.40 & 6.41 & 6.28 & 0.24 \\
\hline LPIN3 & - & - & -- & - & - \\
\hline INSIG1 & 15.43 & 83.21 & 80.89 & 73.04 & 0.89 \\
\hline INSIG2- & 84.56 & 16.78 & 19.10 & 26.95 & 0.48 \\
\hline PPARGC1A & 7.23 & 6.58 & 4.75 & 1.43 & $<0.01$ \\
\hline PPARGC1B- & 92.76 & 93.41 & 95.24 & 98.56 & 0.06 \\
\hline
\end{tabular}

${ }^{a} \mathrm{At}$ each time, mRNA abundance of each isoform in the isoform family was calculated as $\left[\left(\left(1 / \mathrm{E}^{\Delta \mathrm{C}}\right)\right.\right.$ isoform gene/ sum $\left(1 / \mathrm{E}^{\Delta \mathrm{C}}\right)$ isoform gene family $\left.) \times 100\right]$. ' Isoform mRNA abundance among all genes considered in the study was calculated as $\left[\left(\left(1 / \mathrm{E}^{\Delta \mathrm{C}}\right)\right.\right.$ isoform gene/sum $\left(1 / \mathrm{E}^{\Delta \mathrm{C}}\right)$ all genes measured $\left.) \times 100\right]$. For P18, L6, L12, and L18 abbreviations, see legend to Figure 1.

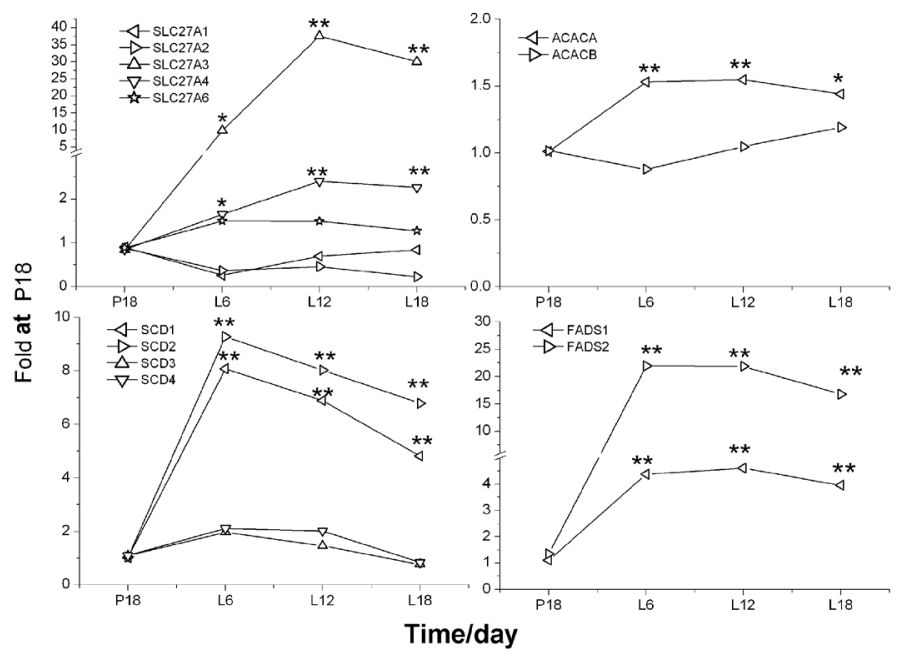

Figure 1. Isoform mRNA expression of SLC27A, ACACA, SCD, and FADS in mouse mammary gland in L6, L12 and L18 (6-, 12- and 18-day lactation). Values are reported as means, for $\mathrm{N}=8$, and are expressed as fold change relative to P18 (18-day pregnance), which was set to $1.0 .{ }^{*} \mathrm{P}<0.05$ compared with $\mathrm{P} 18 .{ }^{*} * \mathrm{P}<0.01$ compared with P18. 
Two isoforms of ACC were discovered in the mouse mammary gland, with ACACA mRNA showing abundance (29.63\%) and ACACB mRNA being almost undetectable $(0.01 \%)$. ACACA was the only isoform with significant upregulation of expression during lactation, while ACACB expression showed no significant difference between lactation and pregnancy (Figure 1). Mammary gland expressed all SCD isoforms, with a high mRNA abundance of SCD1 $(42 \%)$ and SCD2 (17\%). The SCD3 and SCD4 isoforms were barely detectable (Table 1). The mRNA expression of SCD1 and SCD2 increased 10- and 8-fold, respectively (at L6 vs $\mathrm{P} 18, \mathrm{P}<0.01$; Figure 1). FADS1 accounted for $7.56 \%$ of all genes measured, followed by FADS2 (1.58\%), and FADS3 was undetectable (Table 1). FADS1 and FADS2 expression was tremendously upregulated during the transition from the non-lactating period to lactation $(\mathrm{P}<$ 0.01 ; Figure 1). Mouse mammary gland expressed LPIN1 and LPIN2 (2.5 and 0.24\%, respectively, of all genes measured; Table 1), but LPIN3 was undetectable.

Despite these differences, mRNA expression of LPIN1 was upregulated throughout lactation and increased significantly to peak values at L18 $(\mathrm{P}<0.01$; Figure 2$)$.
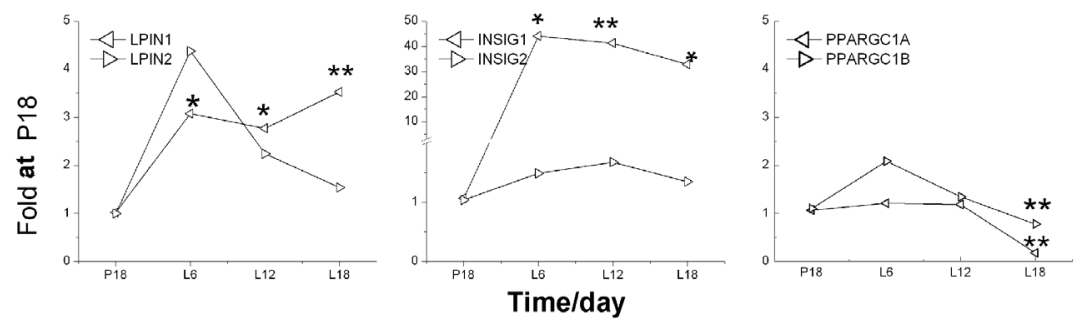

Figure 2. Isoform mRNA expressions of LPIN, INSIG and PPARGC1 in mouse mammary gland in L6, L12 and L18 (6-, 12- and 18-day lactation). Values are reported as means, for $\mathrm{N}=8$, and are expressed as fold change relative to $\mathrm{P} 18$ (18-day pregnance), which was set to 1.0 . $* \mathrm{P}<0.05$ compared with $\mathrm{P} 18$. $* * \mathrm{P}<0.01$ compared with P18.

Mouse mammary gland expressed all isoforms of INSIG and PPARGC1. INSIG1 mRNA expression significantly increased 44-fold at L6 $(\mathrm{P}<0.01)$ and then declined gradually to a 40-fold increase at L12 and a 32-fold increase at L18 $(\mathrm{P}<0.01)$. INSIG2 had lower mRNA abundance $(0.48 \%)$, and its mRNA expression was not affected by the stage of lactation. PPARGC1 was the least abundant isoform ( $0.06 \%$ of all genes measured). PPARGC1A and $1 \mathrm{~B}$ mRNA expressions were significantly downregulated 0.78 - and 0.18 -fold at L18, respectively $(\mathrm{P}<0.01$; Figure 2$)$.

\section{DISCUSSION}

Fatty acid transport proteins (solute carrier family 27, SLC27A) are integral transmembrane proteins that enhance the uptake of fatty acids into cells. Six transporter genes (SLC27A1-6) have been identified in human and mouse genomes (Stahl, 2004). Our results show that isoforms 3 and 4 were the major transporters during the transition from late pregnancy to lactation, while other isoforms with low mRNA abundance and small fold changes appear to have minor functional roles in fatty acid transport in mouse mammary glands.

Unlike in mice, bovine mammary tissue had the most abundant mRNA of SLC27A6 at lactation (Bionaz and Loor, 2008b), suggesting species differences in expression and function 
during mammary TAG synthesis. In the mouse mammary gland, the fold change of SLC27A3 was tremendous between pregnancy and lactation (30-fold). We speculate that during lactation SLC27A3 plays a greater role than SLC27A4 in the uptake of fatty acids from the circulating blood fatty acid pool.

Two isoforms of acetyl-CoA carboxylase (ACACA, ACACB) have been identified in animals (Abu-Elheiga et al., 2000, 2001). Our data clearly point to a major role of ACACA in the metabolic pathway of fatty acid synthesis in the mammary gland, while ACACB appears to have a negligible role. As these modifications in expression of these mRNA isoforms are related to lactation, it would appear that ACACA must relate to a set of unique metabolic requirements for the synthesis of lipids in mouse. Four SCD isoforms (SCD1-4) have been identified in mouse. Our results indicate that SCD1 and 2 are the most important SCD isoforms in the mouse mammary gland and may play an important role in milk fat synthesis. The SCD1 mRNA abundance was the highest (42\%), relative to other classical lipogenic enzyme genes (e.g., ACC and FADS), and the $>8$-fold upregulation during lactation (Figure 2) suggests that it plays a crucial role in TAG synthesis. The reason for the expression of two or more SCD isoforms in the same tissue is not known but seems to be related to the substrate specificity of the isoforms (Ntambi et al., 2004). SCD1 and SCD2 are capable of using 16:0-CoA and 18:0CoA; SCD3 uses C16:0 but not C18:0, and SCD4 uses both C16:0 and C18:0, but the activity is lower than those of other isoforms (Miyazaki et al., 2003). Bovine SCD1 has been recently identified and characterized and can influence milk fatty acid composition (Kgwatalala et al., 2009). It seems that SCD polymorphisms affect the saturation level of milk fat and largely explain the genetic variation in the desaturase indices. In mammals, three isoforms of FADS have been identified (FADS1-3) (Cho et al., 1999a,b; Marquardt et al., 2000). Our results show that FADS1 mRNA expression increased 20-fold at L6 postpartum, whereas FADS2 mRNA increased 4-fold (Figure 2). In the bovine mammary tissue, FADS1 mRNA expression increases 18 -fold by day 60 postpartum, whereas FADS2 mRNA increases only 3 -fold (Bionaz and Loor, 2008a). These data together point to a major role of FADS1 and a minor role of FADS2 in mammary TAG synthesis. LPIN are $\mathrm{Mg}^{2+}$-dependent phosphatidate phosphatase type-1 (PAP1) enzymes, which convert phosphatidate to diacylglycerol (Donkor et al., 2008). There are three members of the LPIN gene family (LPIN1-3), which encode LPIN protein isoforms in mammals (Donkor et al., 2007). At present, very little information exists regarding LPIN isoform function, activity, or expression in the mouse mammary gland. Our data show that mRNA of LPIN1 was the most abundant among the LPIN isoforms and that LPIN1 plays an essential role during lactation. Interestingly, our research found that mRNA expression of LPIN1 was significantly upregulated ( $<0.01$, Figure 2$)$, while LPIN1 mRNA expression of bovine mammary was downregulated at the end of lactation (Bionaz and Loor, 2008a). The results indicate that LPIN1 may have a complicated function in the mouse milk fat synthesis.

A large body of evidence supports the notion that SREBP1 and PPAR $\gamma$ are pivotal in the regulation of mammary lipid synthesis in cows (Harvatine and Bauman, 2006). The specific roles of coactivators of them (e.g., INSIG and PPARGC1) need to be determined. INSIG $(1,2)$ is a key regulator in the processing of SREBPs (Sun et al., 2005). PPARGC1 (A, B) is a transcriptional coactivator that interacts with PPAR $\gamma$, which permits the interaction of this protein with multiple transcription factors (Medina-Gomez et al., 2007). Some research has shown a complex network of coordinated regulatory events in which SREBP, PPAR $\gamma$, INSIG, and PPARGC1 modify the expression of one another (Yabe et al., 2002; Kast-Woelbern et al., 
2004). The mRNA expression of PPARGC1A and PPARGC1B was significantly downregulated at L18, while INSIG was not, suggesting that PPARGC1 may be an important factor in gene regulation.

\title{
ACKNOWLEDGMENTS
}

\author{
Research supported by Chinese National Programs for Science and Technology De- \\ velopment (No. 2006BAD04A03-10).
}

\section{REFERENCES}

Abu-Elheiga L, Brinkley WR, Zhong L, Chirala SS, et al. (2000). The subcellular localization of acetyl-CoA carboxylase 2. Proc. Natl. Acad. Sci. U. S. A. 97: 1444-1449.

Abu-Elheiga L, Matzuk MM, Abo-Hashema KA and Wakil SJ (2001). Continuous fatty acid oxidation and reduced fat storage in mice lacking acetyl-CoA carboxylase 2. Science 291: 2613-2616.

Bernard L, Leroux C and Chilliard Y (2008). Expression and nutritional regulation of lipogenic genes in the ruminant lactating mammary gland. Adv. Exp. Med. Biol. 606: 67-108.

Bionaz M and Loor JJ (2008a). Gene networks driving bovine milk fat synthesis during the lactation cycle. BMC Genomics 9: 366.

Bionaz M and Loor JJ (2008b). ACSL1, AGPAT6, FABP3, LPIN1, and SLC27A6 are the most abundant isoforms in bovine mammary tissue and their expression is affected by stage of lactation. J. Nutr. 138: 1019-1024.

Cho HP, Nakamura MT and Clarke SD (1999a). Cloning, expression, and nutritional regulation of the mammalian Delta-6 desaturase. J. Biol. Chem. 274: 471-477.

Cho HP, Nakamura M and Clarke SD (1999b). Cloning, expression, and fatty acid regulation of the human delta-5 desaturase. J. Biol. Chem. 274: 37335-37339.

Donkor J, Sariahmetoglu M, Dewald J, Brindley DN, et al. (2007). Three mammalian lipins act as phosphatidate phosphatases with distinct tissue expression patterns. J. Biol. Chem. 282: 3450-3457.

Donkor J, Sparks LM, Xie H, Smith SR, et al. (2008). Adipose tissue lipin-1 expression is correlated with peroxisome proliferator-activated receptor alpha gene expression and insulin sensitivity in healthy young men. J. Clin. Endocrinol. Metab. 93: 233-239.

Harvatine KJ and Bauman DE (2006). SREBP1 and thyroid hormone responsive spot 14 (S14) are involved in the regulation of bovine mammary lipid synthesis during diet-induced milk fat depression and treatment with CLA. $J$. Nutr. 136: 2468-2474.

Kast-Woelbern HR, Dana SL, Cesario RM, Sun L, et al. (2004). Rosiglitazone induction of Insig-1 in white adipose tissue reveals a novel interplay of peroxisome proliferator-activated receptor gamma and sterol regulatory element-binding protein in the regulation of adipogenesis. J. Biol. Chem. 279: 23908-23915.

Kgwatalala PM, Ibeagha-Awemu EM, Mustafa AF and Zhao X (2009). Influence of stearoyl-coenzyme A desaturase 1 genotype and stage of lactation on fatty acid composition of Canadian Jersey cows. J. Dairy Sci. 92: 1220-1228.

Marquardt A, Stohr H, White K and Weber BH (2000). cDNA cloning, genomic structure, and chromosomal localization of three members of the human fatty acid desaturase family. Genomics 66: 175-183.

Medina-Gomez G, Gray S and Vidal-Puig A (2007). Adipogenesis and lipotoxicity: role of peroxisome proliferator-activated receptor gamma (PPARgamma) and PPARgammacoactivator-1 (PGC1). Public Health Nutr. 10: 1132-1137.

Miyazaki M, Jacobson MJ, Man WC, Cohen P, et al. (2003). Identification and characterization of murine SCD4, a novel heart-specific stearoyl-CoA desaturase isoform regulated by leptin and dietary factors. J. Biol. Chem. 278: 33904-33911.

Ntambi JM, Miyazaki M and Dobrzyn A (2004). Regulation of stearoyl-CoA desaturase expression. Lipids 39: 1061-1065. Pfaffl MW (2001). A new mathematical model for relative quantification in real-time RT-PCR. Nucleic Acids Res. 29 : e45.

Rudolph MC, McManaman JL, Phang T, Russell T, et al. (2007). Metabolic regulation in the lactating mammary gland: a lipid synthesizing machine. Physiol. Genomics 28: 323-336.

Schwertfeger KL, McManaman JL, Palmer CA, Neville MC, et al. (2003). Expression of constitutively activated Akt in the mammary gland leads to excess lipid synthesis during pregnancy and lactation. J. Lipid Res. 44: 1100-1112.

Stahl A (2004). A current review of fatty acid transport proteins (SLC27). Pflugers Arch. 447: 722-727.

Sun LP, Li L, Goldstein JL and Brown MS (2005). Insig required for sterol-mediated inhibition of Scap/SREBP binding to COPII proteins in vitro. J. Biol. Chem. 280: 26483-26490. 
Vandesompele J, De PK, Pattyn F, Poppe B, et al. (2002). Accurate normalization of real-time quantitative RT-PCR data by geometric averaging of multiple internal control genes. Genome Biol. 3: RESEARCH0034.

Yabe D, Brown MS and Goldstein JL (2002). Insig-2, a second endoplasmic reticulum protein that binds SCAP and blocks export of sterol regulatory element-binding proteins. Proc. Natl. Acad. Sci. U. S. A. 99: 12753-12758.

\section{SUPPLEMENTARY MATERIAL}

Table S1. Primer information for real-time PCR amplification.

\begin{tabular}{|c|c|c|c|c|}
\hline Gene (GenBank number) & Primer sequence & Product length (bp) & Correlation & Efficiency \\
\hline \multirow[t]{2}{*}{ GAPDH (NM001001303) } & F-TGTGTCCGTCGTGGATCTGA & 150 & 0.99 & 1.92 \\
\hline & R-TTGCTGTTGAAGTCGCAGGAG & & & \\
\hline \multirow{2}{*}{ HPRT (NM013556) } & F-CCTAAGATGAGCGCAAGTTGAA & 86 & 0.99 & 2.03 \\
\hline & R-CCACAGGACTAGAACACCTGCTAA & & & \\
\hline \multirow[t]{2}{*}{ RPL13A (NM009438) } & F-AGCGCCTCAAGGTGTTGGA & 168 & 0.98 & 1.96 \\
\hline & R-GAGTGGCTGTCACTGCCTGGTA & & & \\
\hline \multirow[t]{2}{*}{ SLC27A1 (NM011977) } & F-GCAGCATTGCCAACATGGAC & 103 & 0.99 & 2.23 \\
\hline & R-GTGTCCTCATTGACCTTGACCAGA & & & \\
\hline \multirow[t]{2}{*}{ SLC27A2 (NM011978) } & F-AAGTCTCTGCTGCACTGCTTTCA & 186 & 0.99 & 2.34 \\
\hline & R-CGACACTCCGTCTACTTTGTCCA & & & \\
\hline \multirow[t]{2}{*}{ SLC27A3 (NM011988) } & F-TCTGGGACGATTGCCAGAAAC & 116 & 0.98 & 1.93 \\
\hline & R-CAAGCGCACCTTATGGTCACAC & & & \\
\hline \multirow[t]{2}{*}{ SLC27A4 (NM011989) } & F-TGCCCAGTCACCCAGACAAG & 143 & 0.96 & 2.06 \\
\hline & R-CATGCGGAATCCATAGTACACCAG & & & \\
\hline \multirow[t]{2}{*}{ SLC27A6 (NM001081072) } & F-TCTCGCCTCTGATGAGCCAGTA & 156 & 0.94 & 1.87 \\
\hline & R-CATCAGCTGTGCAGCCAAAC & & & \\
\hline \multirow[t]{2}{*}{ ACACA (NM133360) } & F-GAAGTCAGAGCCACGGCACA & 119 & 0.98 & 1.84 \\
\hline & R-GGCAATCTCAGTTCAAGCCAGTC & & & \\
\hline \multirow[t]{2}{*}{ ACACB (NM009128) } & F-CTACAAGACGGCGCAGGTCA & 128 & 0.96 & 2.06 \\
\hline & R-AGGCGCCAAACTTCAGCATC & & & \\
\hline \multirow[t]{2}{*}{ SCD1 (NM009127) } & F-TCTTGTCCCTATAGCCCAATCCAG & 130 & 0.99 & 1.74 \\
\hline & R-AGCTCAGAGCGCGTGTTCAA & & & \\
\hline \multirow[t]{2}{*}{ SCD2 (NM009128) } & F-AGTGTTGCTCGTGAGCCTGTG & 140 & 0.99 & 1.79 \\
\hline & R-CCTGCAGATCCATGTCCAGCTA & & & \\
\hline \multirow[t]{2}{*}{ SCD3 (NM0244502) } & F-TCACACCGTGAACCCTGAGATTGT & 160 & 0.95 & 1.86 \\
\hline & R-TGCTTGCTCTGCCTCTTGACCTAT & & & \\
\hline \multirow[t]{2}{*}{ SCD4 (NM183216.3) } & F-ACCTTGCTCTCTCTGCCTTCACAA & 84 & 0.98 & 1.78 \\
\hline & R-TGCTGGAGATCTCTTGTGGCAAGT & & & \\
\hline \multirow[t]{2}{*}{ FADS1 (NM146094) } & F-TCAGTTTCAGAAGCAGGCAGGA & 86 & 0.99 & 1.88 \\
\hline & R-AAGAATTGCGTGTGCCAGGAC & & & \\
\hline \multirow[t]{2}{*}{ FADS2 (NM019699) } & F-GCCGCTGCTCATCCCTATGTA & 145 & 0.98 & 1.87 \\
\hline & R-GGCTCCCAAGATGCCGTAGA & & & \\
\hline \multirow[t]{2}{*}{ LPIN1(NM172950) } & F-AGGGACACATTTCCACGACAGAG & 114 & 0.96 & 1.98 \\
\hline & R-AAGCCAAGGCTAGGGCTGAGA & & & \\
\hline \multirow[t]{2}{*}{ LPIN2 (NM022882) } & F-AGTCGGAGTTCCAGACTGTAGGATA & 116 & 0.94 & 1.90 \\
\hline & R-CGTGTTCCACAAGCTCACTCA & & & \\
\hline \multirow[t]{2}{*}{ INSIG1 (NM153556) } & F-GCCAGCGTTATGCGCTGTA & 138 & 0.92 & 1.77 \\
\hline & R-GGAACGATCAAATGTCCACCAC & & & \\
\hline \multirow[t]{2}{*}{ INSIG2 (NM133748) } & F-CACTGTCAGTAGGACTGTGGTGGA & 164 & 0.95 & 1.82 \\
\hline & R-TGGCAACCAAGAACGGACATAG & & & \\
\hline \multirow[t]{2}{*}{ PPARGC1A (NM008904) } & F-CCGTAAATCTGCGGGATGATG & 114 & 0.99 & 1.99 \\
\hline & R-CAGTTTCGTTCGACCTGCGTAA & & & \\
\hline \multirow[t]{2}{*}{ PPARGC1B (NM133249) } & F-GTGCCAGGTGCTGACGAGAA & 175 & 0.98 & 1.94 \\
\hline & R-AGTGTATCTGGGCCAACGGAAG & & & \\
\hline
\end{tabular}

Genetics and Molecular Research 9 (2): 1250-1257 (2010) 